\title{
Embryonal Tumor with Multilayered Rosettes, C19MC-Altered
}

National Cancer Institute

\section{Source}

National Cancer Institute. Embryonal Tumor with Multilayered Rosettes, C19MC-Altered. NCl Thesaurus. Code C4915.

An aggressive malignant embryonal neoplasm arising from the central nervous system. It is characterized by the presence of multilayered rosettes formation and the presence of amplification of the C19MC region on chromosome 19 (19q13.42). 